\title{
Metodología de superficie de respuesta dual con variables de ruido cualitativas
}

\section{Dual response surface methodology with qualitative noise variables}

\author{
MARTÍNEZ-MENDOZA, Iván†๋*, VALLES, Adán and SÁNCHEZ-LEAL, Jaime \\ Universidad Tecnológica Paso del Norte, Mexico. \\ Instituto Tecnológico de Ciudad Juárez, Mexico.
}

ID $1^{\text {st }}$ Author: Iván, Martínez-Mendoza / CVU CONACYT ID: 483653

ID $1^{\text {st }}$ Co-author: Adán, Valles / CVU CONACYT ID: 200105

ID $2^{\text {nd }}$ Co-author: Jaime, Sánchez-Leal / CVU CONACYT ID: 61437

DOI: $10.35429 /$ JIE.2020.13.4.13.18

Recibido Julio 25, 2020; Aceptado Diciembre 30, 2020

\section{Resumen}

La aplicación de la metodología de superficie de respuesta en la optimización de procesos industriales ha tenido un gran auge en las últimas décadas, sin embargo, con una limitante significativa, la nula inclusión de factores cualitativos en las variables de ruido. Ya que la metodología asume el comportamiento de los factores de ruido como una variable de comportamiento continua y que sigue una distribución normal. Pero que sucede si no es así, ¿Cómo tratar un factor de ruido cualitativo?, ¿Qué distribución de probabilidad ajustaría de mejor manera al factor de ruido cualitativo?, ¿Cuál sería la correcta inclusión de este tipo de factores de ruido en la metodología?, este articulo resume el trabajo de investigación de cuatro años desde el planteamiento matemático de las nuevas ecuaciones, simulaciones de casos utilizando software matemático y 2 casos reales en plantas maquiladoras que manufacturan piezas de plástico.

Superficie de respuesta, Factor de ruido cualitativo, Optimización de procesos, Distribución de probabilidad

\begin{abstract}
The application of the response surface methodology in the optimization of industrial processes has had a great boom in recent decades, however, with a significant limitation, the null inclusion of qualitative factors in the noise variables. Since the methodology assumes the behavior of the noise factors as a continuous behavioral variable that follows a normal distribution. But what happens if this is not the case? How to treat a qualitative noise factor? What probability distribution would best fit the qualitative noise factor? What would be the correct inclusion of this type of noise factor in the methodology? This article summarizes the four-year research work from the mathematical approach to the new equations, case simulations using mathematical software and 2 real cases in maquiladora plants that manufacture plastic parts.
\end{abstract}

Response surface, Qualitative noise factor, Process optimization, Probability distribution

Citación: MARTÍNEZ-MENDOZA, Iván, VALLES, Adán and SÁNCHEZ-LEAL, Jaime. Metodología de superficie de respuesta dual con variables de ruido cualitativas. Revista de Ingeniería Industrial. 2020. 4-13:13-18.

\footnotetext{
$\dagger$ Investigador contribuyendo como primer autor.
} 


\section{Introducción}

El principal objetivo del diseño robusto consiste en encontrar el nivel óptimo de los factores controlables en un proceso o producto con la finalidad de que los factores de ruido o no controlables no afecten al proceso. Hoy en día las aplicaciones estadísticas para la optimización de procesos son una herramienta útil empleada por la mayoría de las empresas para optimizar la media de sus procesos y disminuir la variabilidad. Con anterioridad se han resuelto problemas de factores robustos empleando el método de superficie de respuesta con factores de ruido cuantitativos, sin embargo, no se tiene registro de problemas resueltos donde los factores de ruido sean cualitativos.

La metodología de superficie de respuesta es un conjunto de técnicas estadísticas y matemáticas utilizadas en el análisis y modelado de problemas en las que una variable de estudio se ve afectada por otras variables. Los orígenes de la metodología de superficie de respuesta (MSR) se remiten al trabajo de Box y Wilson (1951), pero ha sido durante los últimos 20 años que esta metodología ha logrado un desarrollo considerable, tanto en aspectos teóricos como en aplicaciones en escenarios reales. Myers y Montgomery (2009) establecen que la MSR proporciona técnicas estadísticas bien establecidas que se pueden usar para implementar el diseño de parámetros robustos propuesto por Taguchi (1986) y superar sus limitaciones.

El objetivo último de la MSR es determinar las condiciones de operación óptimas del sistema o determinar una región del espacio de los factores en la que se satisfagan los requerimientos de operación (Montgomery, 2005).

Este método, introducido por Myers y Carter (1973), es llamado superficie de respuesta dual donde se puede optimizar la media y simultáneamente minimizar la varianza. Considerando el modelo de superficie de respuesta de segundo grado que incluye un factor de control $(x)$ y uno de ruido cualitativo (z) da como resultado la ecuación 1.

$y=\beta_{0}+\beta_{1} x+\beta_{11} x^{2}+\delta_{1} z+\delta_{11} x z+\varepsilon$
Donde las betas y deltas representan los coeficientes de los factores de control y ruido y la épsilon el error aleatorio del modelo. El factor de ruido es una variable aleatoria de la cual usualmente no se conoce su distribución de probabilidad ni su valor esperado ni su varianza. Aplicando el operador de valor esperado y de la varianza se obtiene las ecuaciones 2 y 3 .

$$
\begin{aligned}
& E(y)=\beta_{0}+\beta_{1} x+\beta_{11} x^{2}+\delta_{1} E(z)+\delta_{11} x E(z) \\
& V(y)=\left(\delta_{1}+\delta_{11} x\right)^{2} V(z)+\sigma^{2}
\end{aligned}
$$

Ya que el valor esperado y la varianza de la variable de ruido $z$ no se conocen, se tienen que estimar a partir de una muestra representativa de sus posibles valores.

El objetivo principal de la investigación presentada en este artículo es desarrollar una metodología que permita optimizar la media y la varianza simultáneamente empleando el método de superficie de respuesta dual en problemas con parámetros robustos que contengan factores de ruido cualitativos y permita aplicar esta metodología en procesos industriales para validar su aplicación exitosa en la optimización de procesos industriales.

La empresa seleccionada para la aplicación de la metodología es la empresa juarense Termoformados de la Frontera ${ }^{\circledR}$, empresa con más de 11 años laborando en el termoformado de charolas y partes de plásticos para empresas como Harman ${ }^{\circledR}$, Robert Bosh ${ }^{\circledR}$, Continental ${ }^{\circledR}$, entre otras.

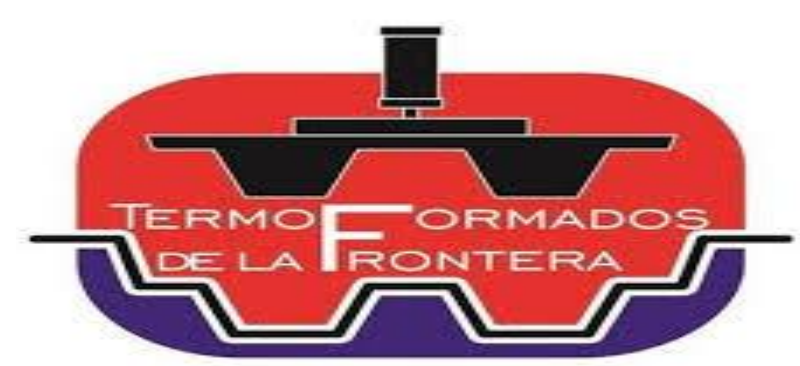

Figure 1 


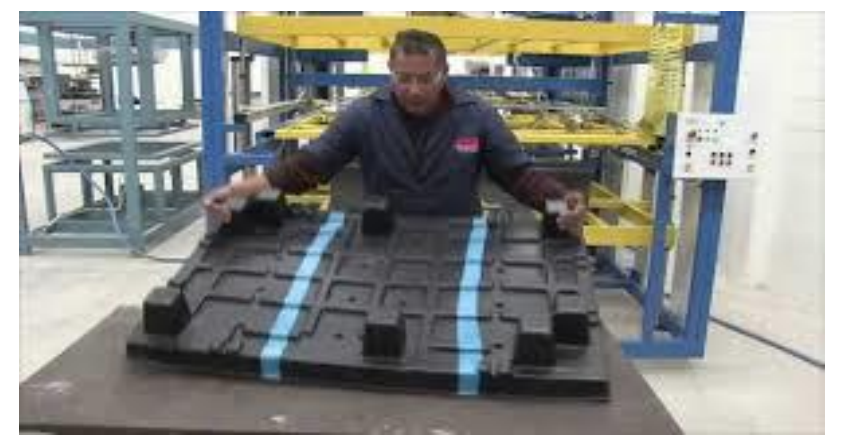

Figure 2

\section{Desarrollo}

Los pasos que se seguirán para definir la metodología de superficie de respuesta dual con variables de ruido cualitativas (MSRDVC) son:

- Definir modelos de la respuesta cuya solución óptima se pueda conocer analíticamente.

- Usar los modelos para simular la respuesta en las corridas experimentales de acuerdo con un diseño experimental apropiado.

- Identificar la distribución de probabilidad de las variables de ruido cualitativas.

- Obtener la solución que optimiza simultáneamente la media y la varianza.

- Comparar la solución obtenida con la metodología MSRDVC con la solución obtenida analíticamente para verificar la efectividad de la metodología MSRDVC.

Validar la metodología MSRDVC en un caso real.

\section{Metodología}

El primer caso consiste en una estación de corte donde entra la charola con núm. de parte 189723 perteneciente al cliente Harman by Samsung ${ }^{\circledR}$, en el cual las charolas termoformadas tienen que ser cortadas y separadas después del proceso de termoformado, debido a que la hoja de plástico que entra a la maquina termo formadora permite en una misma hoja termo formar 2 charolas a la vez, esto por las dimensiones relativamente pequeñas del producto 1 pulgada y media de diámetro.
Para este caso en particular la variable de respuesta de calidad que se medirá es el porcentaje de charolas mal cortadas por cada intervención de los tres distintos empleados, digamos el scrap que cada uno produce al realizar la operación.

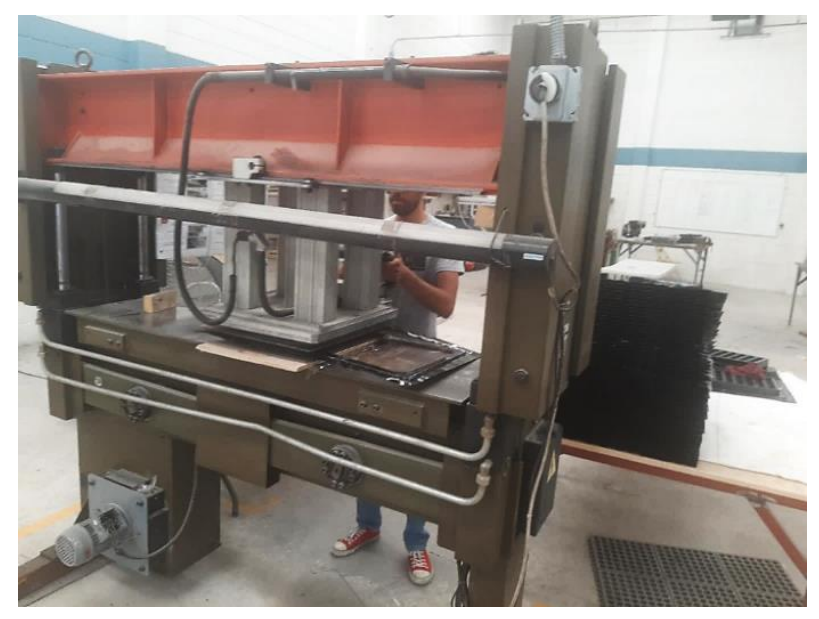

Figura 3 Maquina cortadora de charolas Harman

Para este caso se tomó un factor de control y uno de ruido cualitativo, el factor controlable es la velocidad con la que baja la navaja de la maquina mientras que el factor de ruido cualitativo es el operador en sus niveles: Alex, Ramón y Héctor.

Se procedió a encontrar la ecuación que modelara matemáticamente el proceso de corte y se calculó la siguiente ecuación la cual tiene un máximo en 1.5 lo cual modelara un máximo en la variable de calidad 1.5 pulgadas de diámetro.

$y(x, z)=1+1 x-.5 x^{2}-.5 z+.5 x z$

En la figura 4 se muestra el diseño factorial del proceso de corte para cada corrida experimental correspondiente. Sus tres partes son la factorial $3^{2}$ y sus 3 réplicas. Para evaluar la respuesta se toman muestras durante 3 semanas 5 días a la semana en el único turno que trabaja la cortadora y con los diferentes niveles del factor de ruido (operador). De tal manera que la respuesta $y$ se generó empleando la ecuación 6 y sumándole el error aleatorio simulado. 


\begin{tabular}{|c|c|c|c|c|c|}
\hline StdOrder & RunOrder & PtType & Blocks & velocidad corte & operador \\
\hline 9 & 1 & 1 & 1 & 3 & 3 \\
\hline 3 & 2 & 1 & 1 & 1 & 3 \\
\hline 13 & 3 & 1 & 1 & 2 & 1 \\
\hline 25 & 4 & 1 & 1 & 3 & 1 \\
\hline 11 & 5 & 1 & 1 & 1 & 2 \\
\hline 23 & 6 & 1 & 1 & 2 & 2 \\
\hline 12 & 7 & 1 & 1 & 1 & 3 \\
\hline 19 & 8 & 1 & 1 & 1 & 1 \\
\hline 26 & 9 & 1 & 1 & 3 & 2 \\
\hline 2 & 10 & 1 & 1 & 1 & 2 \\
\hline 16 & 11 & 1 & 1 & 3 & 1 \\
\hline 24 & 12 & 1 & 1 & 2 & 3 \\
\hline 15 & 13 & 1 & 1 & 2 & 3 \\
\hline 7 & 14 & 1 & 1 & 3 & 1 \\
\hline 27 & 15 & 1 & 1 & 3 & 3 \\
\hline 5 & 16 & 1 & 1 & 2 & 2 \\
\hline 6 & 17 & 1 & 1 & 2 & 3 \\
\hline 4 & 18 & 1 & 1 & 2 & 1 \\
\hline 22 & 19 & 1 & 1 & 2 & 1 \\
\hline 20 & 20 & 1 & 1 & 1 & 2 \\
\hline 10 & 21 & 1 & 1 & 1 & 1 \\
\hline 18 & 22 & 1 & 1 & 3 & 3 \\
\hline
\end{tabular}

Figura 4 Diseño Factorial Minitab®

Después de obtener por medio del muestreo la respuesta correspondiente a cada corrida experimental del diseño factorial se introduce la respuesta en el software Minitab® para obtener un modelo de regresión lineal. Se procede a generar empleando Minitab ${ }^{\circledR}$ el modelo de regresión lineal que se ajuste a los datos obtenidos de la respuesta. Luego, a partir de este modelo general, se obtienen las superficies de respuesta tanto para la media como la varianza.

El siguiente paso consiste en identificar la distribución a la que mejor se ajustan los datos del factor de ruido, para nuestro caso se asumió que el comportamiento del factor de ruido sería una distribución de probabilidad uniforme discreta. Para esto se llevó a cabo el muestreo del factor de ruido. En la figura 5 se muestra la tabla donde se realizó el muestreo de los valores que representan los niveles del factor de ruido empleando el software Excel®.

En la primera parte podemos observar la simulación del factor de ruido generando un numero aleatorio entre 0 y 1 y después se asignan tres posibles diferentes niveles para el factor, nivel -1 si el numero está entre 0 y .33 correspondiente a Ramon, nivel 0 si esta entre 0.34 y .66 correspondiente a Alex y por último el nivel 1 si esta entre .67 y 1 correspondiente a Hector.
Además, el macro en Excel incluye una serie de graficas que nos permite observar la aleatoriedad para el factor de ruido en la primera gráfica y el comportamiento de la media y desviación estándar, cabe destacar que solo son 18 muestras por día debido a que la operación de corte se hace cada 30 minutos en el turno para evitar acumulación de charolas, el turno tiene 9 horas operativas y se realizan 18 operaciones de corte, también es importante comentar que el empleado desocupado acciona la operación. Con la media en 1.83 podríamos decir que el empleado Alex es el que más seguido la ópera en un turno.

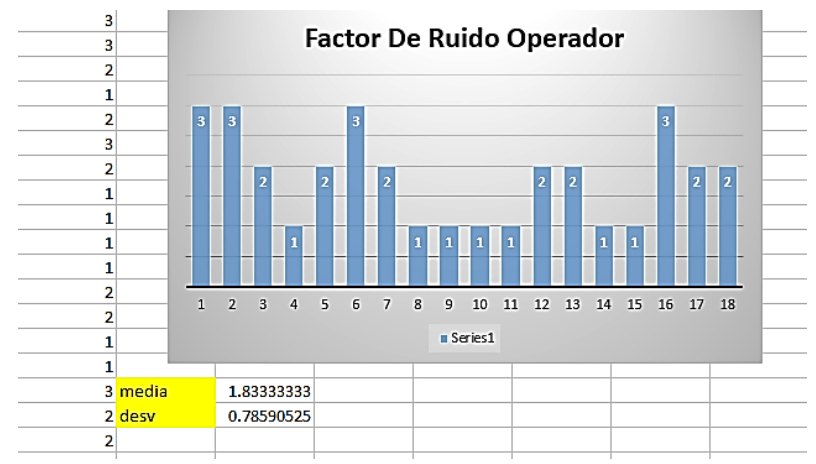

Figura 5 Factor de ruido Cualitativo Excel ${ }^{\circledR}$

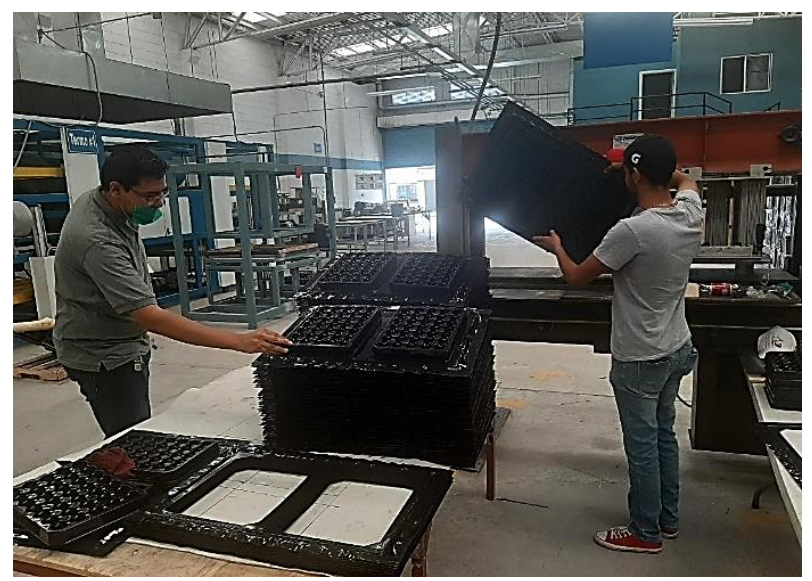

Figura 6 Proceso de Corte

\section{Resultados}

Una vez obtenidos el modelo general en el software Minitab® y la media y varianza del factor de ruido se emplea el software matemático Matlab ${ }^{\circledR}$ que permite optimizar los modelos de primer y segundo grado que se obtienen al hacer el análisis de superficie de respuesta dual y encontrar el máximo y el peso óptimo de la media. Además del criterio simultaneo y el peso optimo ideal, el criterio simultaneo permite optimizar juntos la media y varianza en el modelo para cada una de las quince simulaciones $\mathrm{y}$ al final los totales. 
Los resultados con un factor de control y un factor de ruido cualitativo con un comportamiento de una distribución uniforme se muestran en la tabla 1, donde se muestra los resultados de 15 corridas en la tabla 1. En las primeras dos columnas se incluyen la media del factor de ruido $E(z)$ y varianza simulada $V(z)$ y en las restantes se muestran el valor máximo de la media y el valor mínimo de la varianza al optimizarse de manera independiente.

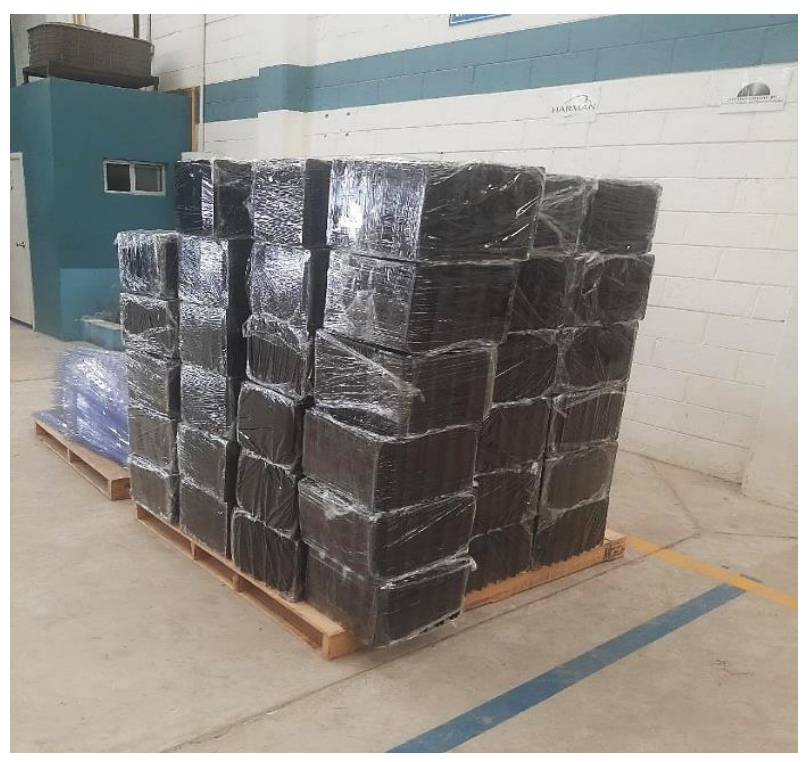

Figura 7 Producto terminado

\begin{tabular}{|c|c|c|c|c|c|}
\hline$z$ & $V z$ & $E(\max )$ & $V(\mathrm{~min})$ & max simultaneo & peso opt \\
\hline 0.008 & 0.06804 & 1.45662 & 0.21227 & 1.45642 & $1 / 9500$ \\
\hline-0.001 & 0.01 & 1.41411 & 0.19997 & 1.41399 & $1 / 12000$ \\
\hline 0.005 & 0.06944 & 1.63876 & 0.26855 & 1.63858 & $1 / 9000$ \\
\hline-0.003 & 0.0658 & 1.63882 & 0.26857 & 1.63862 & $1 / 8000$ \\
\hline 0 & 0.06868 & 1.3286 & 0.17652 & 1.32832 & $1 / 7500$ \\
\hline 0.013 & 0.07405 & 1.5537 & 0.2414 & 1.5536 & $1 / 5000$ \\
\hline 0.006 & 0.0562 & 1.58682 & 0.2518 & 1.58665 & $1 / 5000$ \\
\hline 0.004 & 0.06852 & 1.62304 & 0.26342 & 1.6229 & $1 / 5000$ \\
\hline 0.007 & 0.06516 & 1.474888 & 0.21753 & 1.47471 & $1 / 9000$ \\
\hline 0.004 & 0.0665 & 1.47906 & 0.21876 & 1.47889 & $1 / 8500$ \\
\hline 0.008 & 0.05793 & 1.44536 & 0.20891 & 1.44518 & $1 / 8500$ \\
\hline 0.005 & 0.06944 & 1.53928 & 0.23694 & 1.53911 & $1 / 9000$ \\
\hline-0.004 & 0.05842 & 1.44133 & 0.20774 & 1.4117 & $1 / 9000$ \\
\hline 0.008 & 0.07208 & 1.47649 & 0.218 & 1.47632 & $1 / 8500$ \\
\hline 0.012 & 0.05915 & 1.5649 & 0.24489 & 1.56467 & $1 / 9000$ \\
\hline 0.0048 & 0.0661927 & 1.510785 & 0.2290113 & 1.5106006667 & $1 / 11250$ \\
\hline
\end{tabular}

Tabla 1 Resumen de Resultados
Se verifica la cercanía de la respuesta con el valor ideal de 1.5 pulgadas, con el fin de validar la funcionalidad de la metodología, la respuesta óptima es un diámetro de 1.5 pulgadas, si comparamos el valor de 1.51060 el error total porcentual seria del $.7 \%$, aunado a una varianza promedio de .29 que relativamente es un valor bajo. Y dado que los límites de especificación de la charola es 1.5 pulgadas más menos .12 pulgadas estamos dentro de los limites especificados.

\section{Conclusiones}

Como se puede observar en la tabla de resumen de resultados en promedio todos los resultados se acercaron bastante al valor óptimo de respuesta, lo cual muestra resultados muy cercanos al valor óptimo de la media de 1.5 pulgadas y una varianza promedio de .22 pulgadas. Respecto a los resultados observados en la aplicación para la optimización de la operación de corte empleando la superficie de respuesta dual en un caso real en la empresa Termoformados de la Frontera ${ }^{\circledR}$ con el propósito de verificar que la metodología resuelva distintos casos que se puedan presentar en la práctica de los procesos industriales.

También cabe destacar que con los resultados previstos en esta investigación desde los primeros resultados dados por medio de simulación nos da la idea de que la mejor manera de introducir el factor de ruido cualitativo en la superficie de respuesta dual seria asumiendo el supuesto de que el factor de ruido sigue una distribución uniforme discreta y que es útil el método Montecarlo $(-1,0,1)$ para representar los niveles, siempre y cuando este tenga 3 niveles.

\section{Referencias}

Box y Wilson (1951) Introducción a la Metodología de Superficie de Respuesta, la revista de la Royal Statistical Society

Myers R. y Montgomery D. (2009), Response Surface Methodology, New York

Myers R. y Montgomery D. (1995), Response Surface Methodology, New York

Myers R. Y Carter (1973), Response Surface Techniques for dual response 
Montgomery D. (2005), Diseño y análisis de experimentos. Editorial Limusa 2da Ed. pp 1

Taguchi, G. (1986) Introduction to Quality Engineering: Designing Quality into Products and Processes. Asian Productivity Organization, Tokyo. 